\title{
Article \\ Ultra-Wideband MIMO Array for Penetrating Lunar Regolith Structures on the Chang'e-5 Lander
}

\author{
Wei Lu ${ }^{1,2,3}$, Yuxi Li ${ }^{1,2}$, Yicai Ji ${ }^{1,2,3, *}$, Chuanjun Tang ${ }^{1,2}$, Bin Zhou ${ }^{1,2}$ and Guangyou Fang ${ }^{1,2,3}$ \\ 1 Aerospace Information Research Institute, Chinese Academy of Sciences, Beijing 100190, China; \\ luwei@aircas.ac.cn (W.L.); liyx002994@aircas.ac.cn (Y.L.); tangcj@aircas.ac.cn (C.T.); \\ zhoubin@aircas.ac.cn (B.Z.); gyfang@mail.ie.ac.cn (G.F.) \\ 2 Key Laboratory of Electromagnetic Radiation and Sensing Technology, Chinese Academy of Sciences, \\ Beijing 100190, China \\ 3 School of Electronic, Electrical and Communication Engineering, University of Chinese Academy of Sciences, \\ Beijing 100049, China \\ * Correspondence: ycji@mail.ie.ac.cn
}

check for updates

Citation: Lu, W.; Li, Y.; Ji, Y.; Tang, C.; Zhou, B.; Fang, G. Ultra-Wideband MIMO Array for Penetrating Lunar Regolith Structures on the Chang'e-5 Lander. Electronics 2021, 10, 8. https://dx. doi.org/10.3390/electronics10010008

Received: 18 November 2020 Accepted: 21 December 2020 Published: 23 December 2020

Publisher's Note: MDPI stays neutral with regard to jurisdictional claims in published maps and institutional affiliations.

Copyright: (C) 2020 by the authors. Licensee MDPI, Basel, Switzerland. This article is an open access article distributed under the terms and conditions of the Creative Commons Attribution (CC BY) license (https: / / creativecommons.org/ licenses/by/4.0/).

\begin{abstract}
The Chang'e-5 lunar exploration mission of China is equipped with a Lunar Regolith Penetrating Radar (LRPR) for measuring the thickness and structures of the lunar regolith in the landing area. Since the LRPR is stationary, an ultra-wideband multiple-input multiple-output (MIMO) array is designed as a replacement for conventional mobile subsurface probing systems. The MIMO array, with 12 antenna elements and a switch matrix, operates in the frequency band from 1.0 to $4.75 \mathrm{GHz}$. In this work, the design and layout of the antenna elements were optimized with respect to the lander. To this end, the antenna elements were designed as miniaturized Vivaldi antennas with quarter elliptical slots (i.e., quarter elliptical slotted antenna, or QESA). QESAs are significantly small while being able to mitigate the impact of the lander on antenna electrical performances. QESAs also have a wide operating bandwidth, flat gain, and excellent time domain characteristics. In addition, a high-temperature resistant ultra-light radome with high transmissivity is designed to protect the external antenna array. After calibration, the MIMO array is used to detect targets embedded in volcanic ash. The detection depth reaches $2.5 \mathrm{~m}$, and the detection effect is good.
\end{abstract}

Keywords: MIMO array; vivaldi antenna; ultra-wideband; Chang'e-5; LRPR

\section{Introduction}

At the end of 2020, China will conduct the Chang'e-5 (CE-5) lunar exploration mission. The Chang'e-5 lander is equipped with a Lunar Regolith Penetrating Radar (LRPR). The LRPR is a high-resolution lunar regolith probing imaging radar for detecting the thickness and fine structure of the lunar regolith in the landing area. The radar is used for studying the structure, electromagnetic characteristics and mineral composition of lunar regolith, and analyzing the formation and evolution of lunar regolith. The structure information of lunar regolith detected by LRPR can support the work of drilling and sampling.

Since the 1970s, the Apollo Lunar Sounder Experiment (ALSE) on the Apollo-17 and Lunar Radar Sounder (LRS) onboard the SELENE have successively explored the subsurface structure of the moon in lunar orbit [1,2]. In 2012 and 2018, the Lunar Penetrating Radar (LPR), carried by Chang'e-3 and Chang'e-4, implemented high-resolution sounding on the surface of the lunar with a depth of hundreds of meters [3,4]. The Mars Penetrating Radar (MPR) system onboard Tianwen-1 mission of China and the Radar Imager for Mars' Subsurface Exploration (RIMFAX) system for National Aeronautics and Space Administration's (NASA) Mars 2020 rover mission will be detecting on the surface of Mars in 2021 [5,6]. All of the above radar systems are satellite or rover borne systems and detected by mobile scanning. Since the CE-5 lander is stationary, it is necessary to design a new sub-surface probing mode to replace the conventional mode. For this reason, an 
ultra-wideband multiple-input multiple-output (MIMO) array is designed, which performs two-dimensional scans of the area below the array by using a switch matrix to switch antennas between the transmitting and receiving states. This is the first application of this method in aerospace, and there is almost no ground-based precedent, except that Motoyuki Sato has used a similar method [7].

The proposed MIMO array consists of 12 elements which uses miniature quarter elliptical slotted Vivaldi antennas (QESAs), with the thoroughly optimized placements on the CE-5 lander. This design reduces the impact of the lander on antenna performance and ensures that the standing wave ratio (SWR) of the antenna elements is $<2.0$ throughout the range of 1.0-4.75 GHz. QESAs are capable of providing 2-8 dBi of realized gain and there are no grating lobes in the operating band. The length and width of the QESA are both one-third the wavelength of the lowest operating frequency, which is significantly smaller than conventional Vivaldi antennas [8]. The MIMO array uses a switch to set an antenna element in the transmitting or receiving state, one of the 12 elements transmits the impulses, and the remaining elements receive the echo signals at the same time. In one full scan, each element will be transmitted one-by-one, forming 132 traces of data. In addition, three radomes are designed to ensure that the array can withstand the temperature from -150 to $+250{ }^{\circ} \mathrm{C}$ and protect the antennas from the pollution of lunar dust.

Section 2 introduces the design requirements and application requirements of the antenna array. Section 3 describes the design of the antenna element and the array, and some simulation and measurement results are shown. In the Section 4, the calibration and test verification methods of antenna array are given, and the effectiveness of calibration is demonstrated. The detection effect of the array is verified by testing. Sections 5 and 6 provide the discussion and conclusion.

\section{Antenna Design Requirements}

The design requirements of MIMO array mainly come from the scientific objectives of LRPR and mission requirements. The operating frequency of LRPR is defined from 1.0 to $4.75 \mathrm{GHz}$, because LRPR is necessary to detect the structure of lunar regolith within $2.5 \mathrm{~m}$ thickness and achieve the depth resolution of a few centimeters. Since the CE- 5 lander is stationary, which is different from all previous subsurface structure exploration on Lunar or Mars, the LRPR antenna system adopts MIMO array and replaces the traditional detection mode by electrical scanning. The array is divided into three groups, which are respectively installed at the bottom and side of the lander. The radiation direction of each element is ensured to point to the lunar surface. The bottom structure of the lander is complex and the coupling between the antenna and the lander is strong, which changes the radiation characteristics and impedance characteristics of the antenna. Therefore, it is necessary to optimize the element design and array layout combined with the lander.

The installation position of the array is only $0.7 \mathrm{~m}$ away from the landing engine. During the landing process, part of the plume generated by the engine directly diffuses to the array position, and part of the plume is reflected to the antenna position by the lunar surface. The plume will heat the array rapidly. By the thermal analysis, the maximum temperature of the antenna may reach $250^{\circ} \mathrm{C}$, so the MIMO array should be able to adapt the temperature from -150 to $+250^{\circ} \mathrm{C}$. The main design indexes of the MIMO array are listed in Table 1. 
Table 1. Multiple-input multiple-output (MIMO) array design requirements.

\begin{tabular}{cc}
\hline Parameter & Value \\
\hline Operating band & $1.0-4.75 \mathrm{GHz}$ \\
Gain & $\mathrm{G}_{\mathrm{LF}} \geq 1 \mathrm{dBi} \mathrm{G}_{\mathrm{HF}} \geq 6 \mathrm{dBi}$, increase with frequency \\
Polarization & $\leq-10 \mathrm{~dB}$ \\
Match (S11) & $\geq 50^{\circ}$, direct to lunar surface \\
Beam width & -150 to $+250{ }^{\circ} \mathrm{C}$ \\
Temperature range & $\leq 1.6 \mathrm{~kg}$ \\
Total mass & Group A: $800 \times 120 \times 120 \mathrm{~mm}^{3}$ \\
& Group B: $300 \times 240 \times 120 \mathrm{~mm}^{3}$ \\
Volume & Group C: $120 \times 60 \times 120 \mathrm{~mm}^{3}$ \\
\hline
\end{tabular}

\section{MIMO Array System Design}

\subsection{Antenna Element Design}

The width and length of a conventional Vivaldi antenna are generally not less than $\lambda / 2$ and $2 \lambda$, respectively $[9,10]$. Since conventional antenna designs cannot meet the strict weight and volume requirements of space exploration, a miniaturized antenna must be designed specifically for this purpose. If the miniaturization problem is addressed in isolation, these weight and volume requirements may be met using current techniques for the miniaturization of Vivaldi antennas $[8,11]$. The electrical performance of the antenna is deteriorated due to the strong coupling between the antenna and the lander which makes the existing methods difficult to satisfy the mission requirements. Therefore, the impact of the lander should be properly considered in the design.

The QESA design was developed on the basis of analyses on how the array configuration and lander affect antenna performance. The QESAs are made of ceramic filled polyimide laminate with relative permittivity of 3.8, loss tangent of 0.006 and thickness of $1 \mathrm{~mm}$. The structure of the QESA is shown in Figure 1. The tapering lines represent the shape of the metallic edge of the radiating area, which can be mathematically expressed as Formulas (1) and (2) [12].

$$
\begin{gathered}
y= \pm\left(\frac{w}{2} \times \frac{e^{\alpha t}-1}{e^{\alpha}-1}+\frac{w_{\text {throat }}}{2}\right) \\
z=\left(l-d-l_{1}-l_{\text {throat }}\right) \times t
\end{gathered}
$$

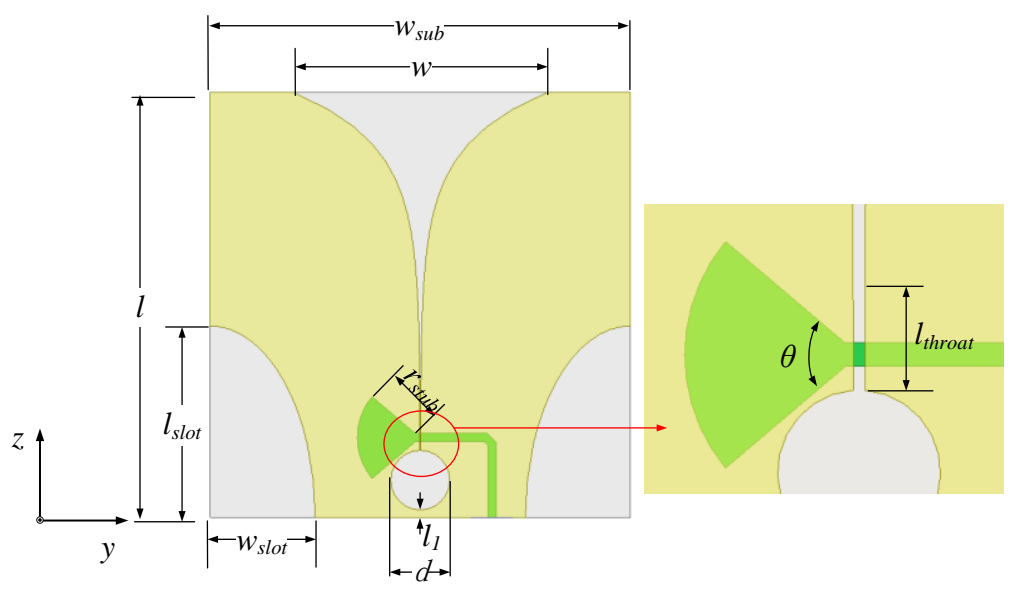

Figure 1. Geometry of the quarter elliptical slotted antenna (QESA) The yellow area shows the copper on the top layer, the green area shows the copper on the bottom layer. The structural parameters of the QESA are as follows: $w_{\text {sub }}=l=100 \mathrm{~mm}, w=60.8 \mathrm{~mm}, l_{\text {slot }}=40 \mathrm{~mm}, w_{\text {slot }}=25 \mathrm{~mm}, d=14 \mathrm{~mm}$, $l_{\text {throat }}=4.27 \mathrm{~mm}, r_{\text {stub }}=15 \mathrm{~mm}, \theta=80^{\circ}$, and $l_{1}=1.7 \mathrm{~mm}$. 
In these equations, $t$ varies from 0 to 1 , and $\alpha=6.2$ defines the taper profile in $y$ direction. According to Figure 1, $l$ is the total length of the antenna along axis $z$, $w_{\text {sub }}$ is the total width of the antenna along axis $y, w_{\text {throat }}$ is the gap between the two tapered curves at the feed point, and $l_{\text {throat }}$ is the length of parallel line at the feed point, $l_{\text {slot }}$ and $w_{\text {slot }}$ are the sizes of the quarter elliptical slots which are symmetrically distributed on both sides of the antenna.

The QESA uses a microstrip-to-slotline transition as its feed. To create a broadband matching antenna, the slotline and microstrip terminals were designed as wideband short stub and open stub, respectively. The characteristic impedances of the slotline and microstrip are 71 and $50 \Omega$.

\subsection{Array Design}

The MIMO antenna array consists of 12 elements. To ensure that the MIMO array would be able to image the drilling site, the elements layout was optimized such that all elements were installed around the driller. Elements 1-10 were placed on the sides of the drilling mechanism, while elements 11 and 12 were placed close to the mechanism to enhance the imaging capability. All the elements maintain the same polarization and form array along the H-plane. The distance between two adjacent antennas is $120 \mathrm{~mm}$. All of the antennas' main beams were directed at the lunar surface. The layout of the antenna elements is shown in Figure 2. The 12 elements were divided into three groups: Group A, B, and C. Groups A and B were installed on the bottom of the lander, while Group C was installed on the side of the lander. The MIMO array has a switch matrix that switches the elements between their transmitting and receiving states. In a full scan, each of the 12 elements will transmit, one after another, while the 11 remaining elements act as receivers to receive echo signals. In this way, 132 traces of valid data will be formed, thus allowing the MIMO array to probe the structure of the lunar regolith beneath the lander.

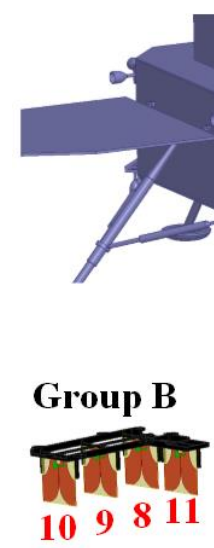

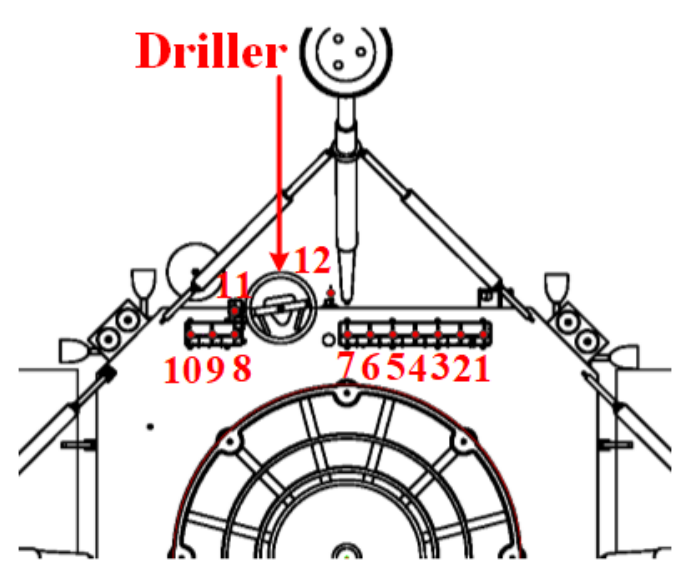

(b)

Figure 2. Layout of the MIMO array. (a) Oblique view. (b) Bottom view.

In the process of lunar landing, the plume of the engine may heat the antenna to $250{ }^{\circ} \mathrm{C}$. At the same time, the plume will stir up charged lunar dust. If a large amount of lunar dust adheres to the elements, the performance of the antenna will be affected. In order to protect the array, ultra-light radomes with high transmission rate are designed for each group of elements. The radomes are made of polyimide composite with a density of $1.8 \mathrm{~g} / \mathrm{cm}^{3}$ and temperature resistance of $350{ }^{\circ} \mathrm{C}$. In the $1-5 \mathrm{GHz}$ frequency band, the relative permittivity of the radome is between 3.6 and 4.2 , and the loss tangent is between 0.012 and 0.016. The test results are shown in Figure 3. The dielectric properties were measured by the syntonic cavity method. In the production of the radomes, three standard size test pieces with size of $\varnothing 162 \times 3 \mathrm{~mm}$ were produced at the same time with the same raw materials and techniques. The test error is better than $10 \%$ in the $1-1.7 \mathrm{GHz}$ band and better 
than $5 \%$ in the $1.7-5 \mathrm{GHz}$ band. Through the mechanical analysis, the radome is designed as a $0.6 \mathrm{~mm}$ thick thin-walled cavity structure, and the lightweight design is realized. The transmission rate of the radome is tested, and the result is better than $97 \%$. The measured results are shown in Figure 3. The radome can effectively isolate the lunar dust and prevent the engine plume from directly splashing onto the elements, which reduces the heating rate of the array. In order to verify the protective effect of the radome, an engine plume test was carried out. The array was installed on a plate to simulate the real installation position. The test device was placed in a vacuum tank, and the ambient temperature was $-60{ }^{\circ} \mathrm{C}$. The engine ignited in pulse mode for $3 \mathrm{~s}$. The temperature response data is shown in Figure 4 . When the engine works for a short time, the maximum temperature of the core components of the array is effectively reduced, and the temperature adaptability of the array is improved. In order to verify the extreme temperature tolerance of the array, a $250{ }^{\circ} \mathrm{C}$ storage test was carried out. The high temperature lasted for $30 \mathrm{~min}$. After the test, the color of the radome and elements changed slightly, but the electrical properties remained. The MIMO array with the radomes installed is shown in Figure 5.

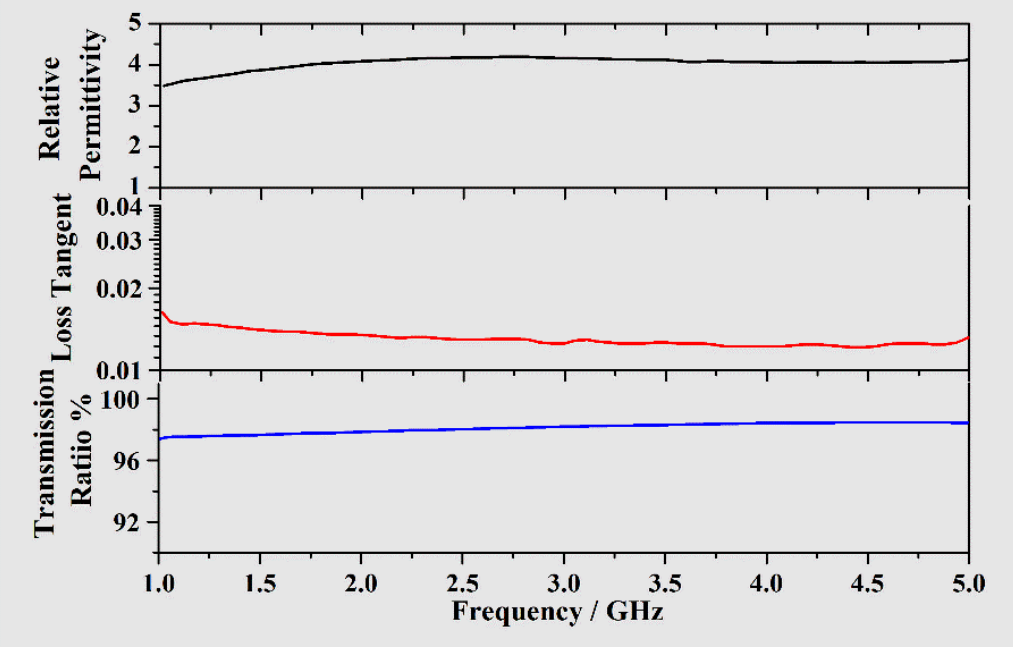

Figure 3. Electromagnetic characteristics of the radome.

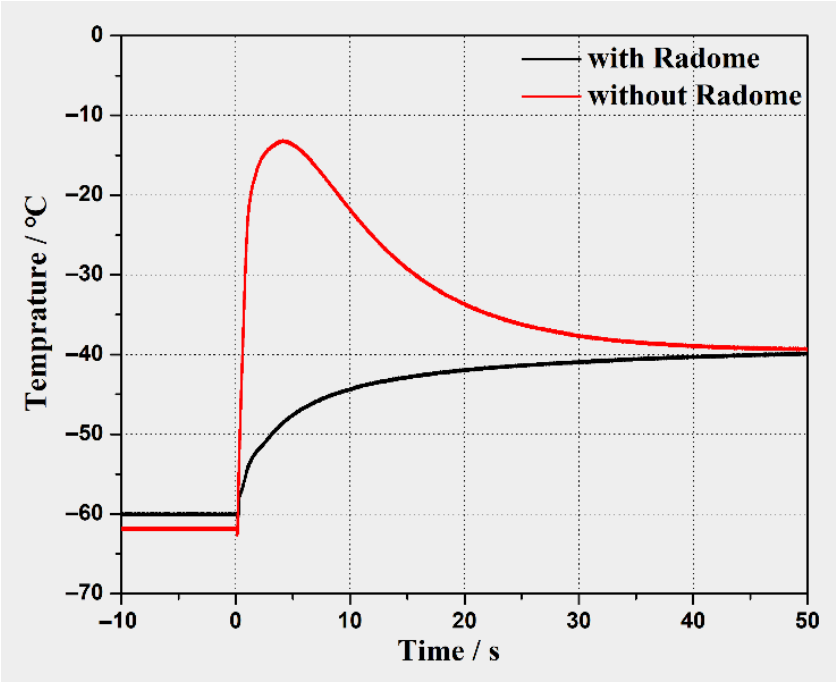

Figure 4. Temperature response of the array. 


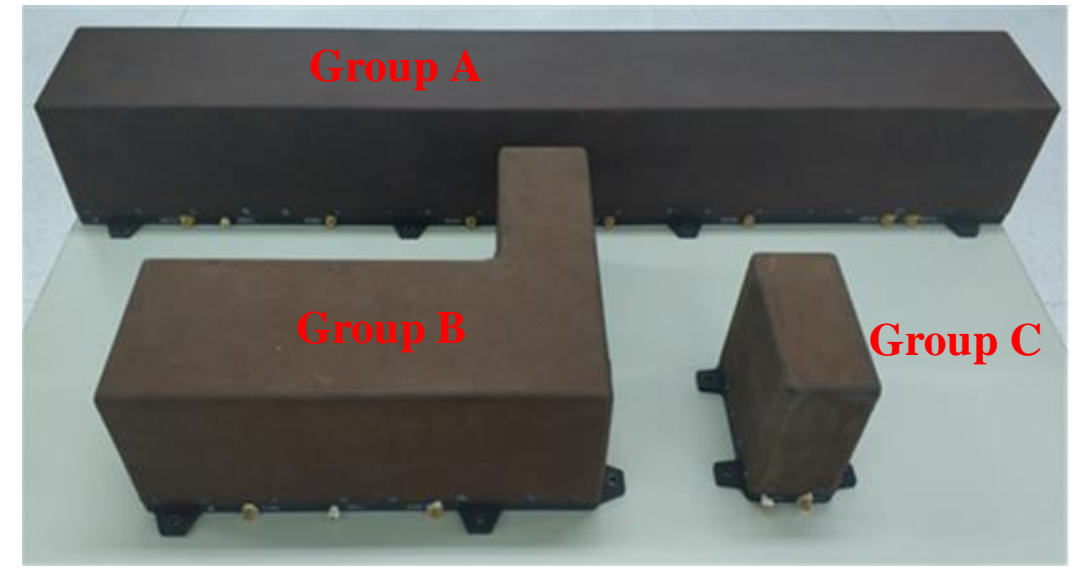

Figure 5. Photograph of the MIMO array.

\subsection{Simulation and Measurement}

As displayed in Figure 6, whether it is an individual antenna or an element in the array, the S11 shows excellent performance in the range of 1.0 to $4.75 \mathrm{GHz}$, which ensures that the antenna will not produce impedance mismatch in the array. The test status and results of the QESA are shown in Figures 7 and 8, respectively. The realized gain fluctuates between 2 and $8 \mathrm{dBi}$ through the measurement of the QESA in a microwave anechoic chamber. By and large, the gain of QESA increases with the frequency.

It can be observed from Figure 9 that the simulated and measured forward radiation patterns of the antenna are in good agreement. In order to reduce the influence of the turntable, the absorber is used during the test to block the mechanism which results in obvious difference between the measured and simulated backward radiation. When the QESA was excited by a monocycle pulse with width of $0.5 \mathrm{~ns}$, it exhibited an excellent waveform with a short tail (see Figure 10).

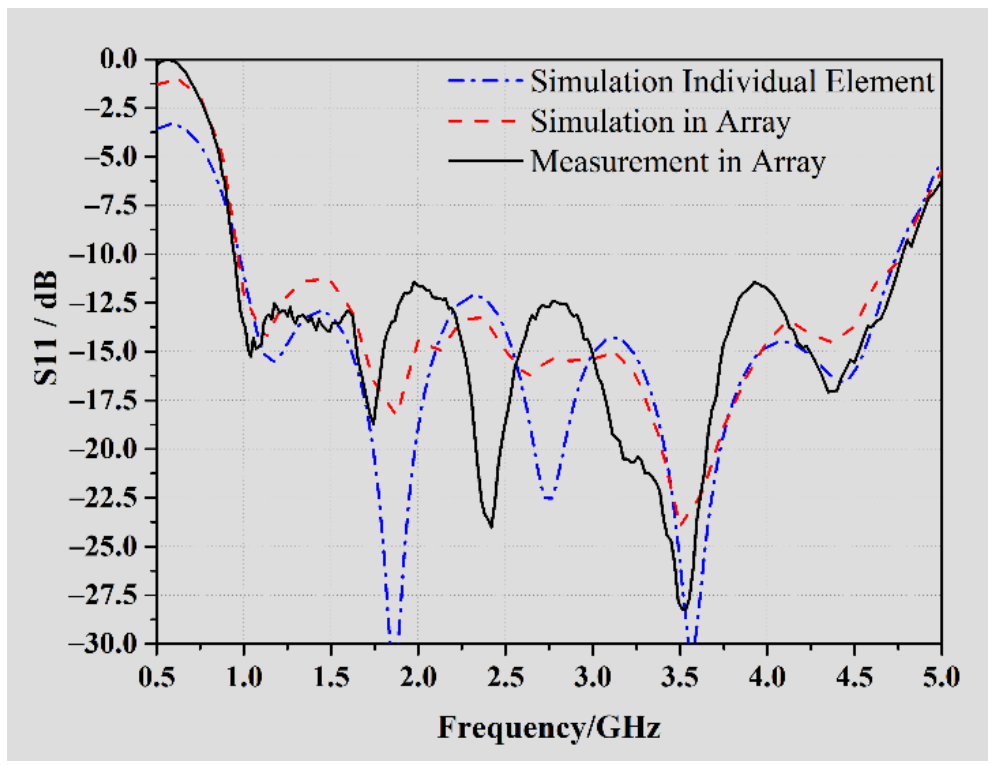

Figure 6. Simulated and measured S11 of QESA mounted on the lander (S11). The blue dash line is the simulated result of an individual antenna, the red dash line is the simulated result of an element in the array, the black solid line is the measured result of an element in the array. 


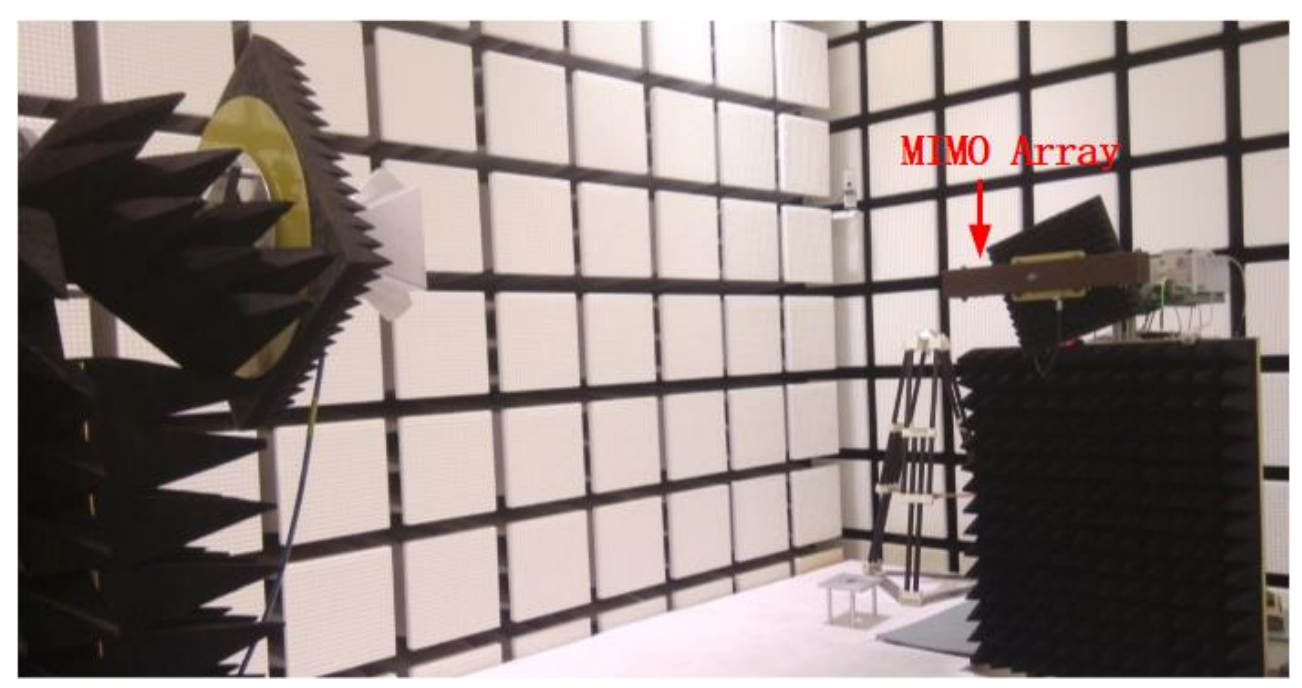

Figure 7. Status of the antenna test in anechoic chamber. The antenna under test is the fourth element of group A.

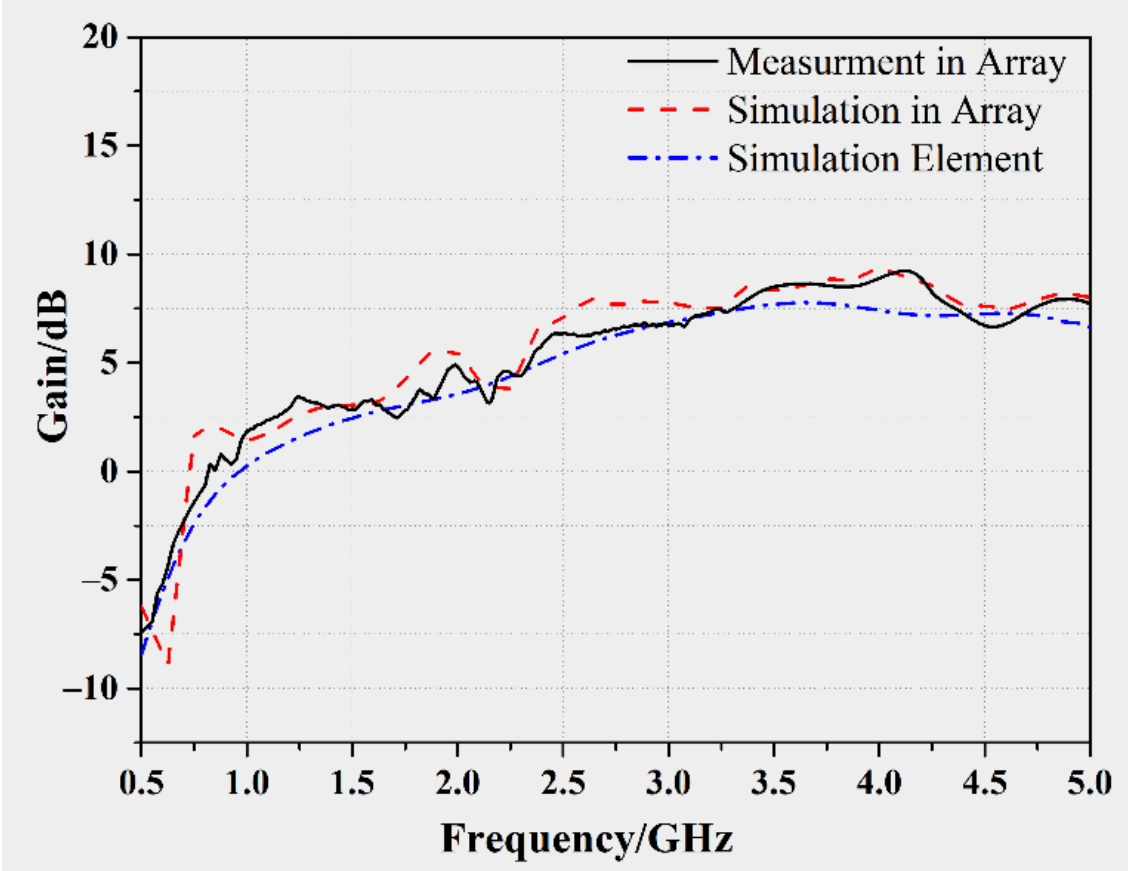

Figure 8. Comparison of realized gains between simulation and measurement. 


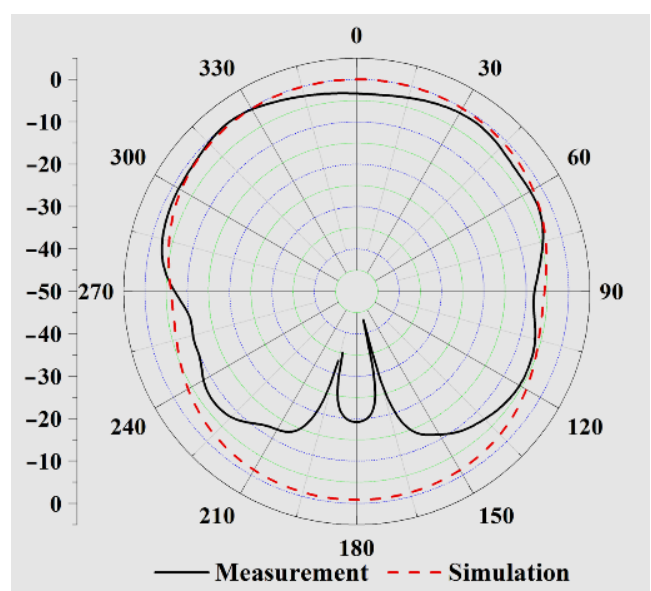

(a)

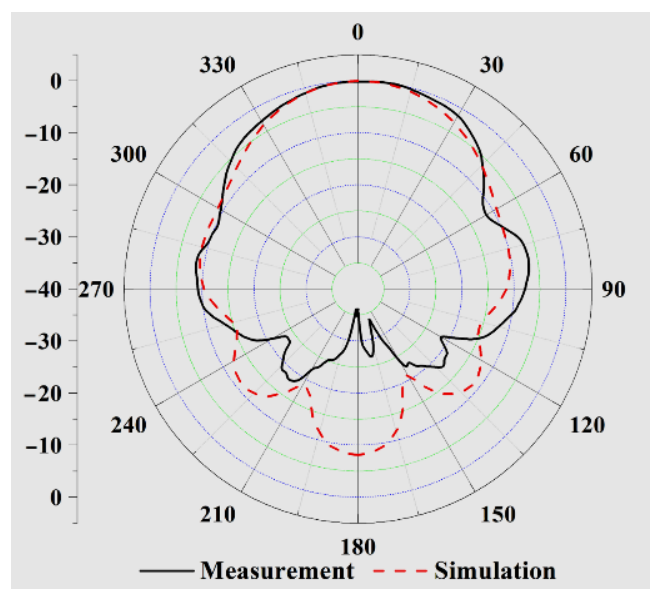

(c)

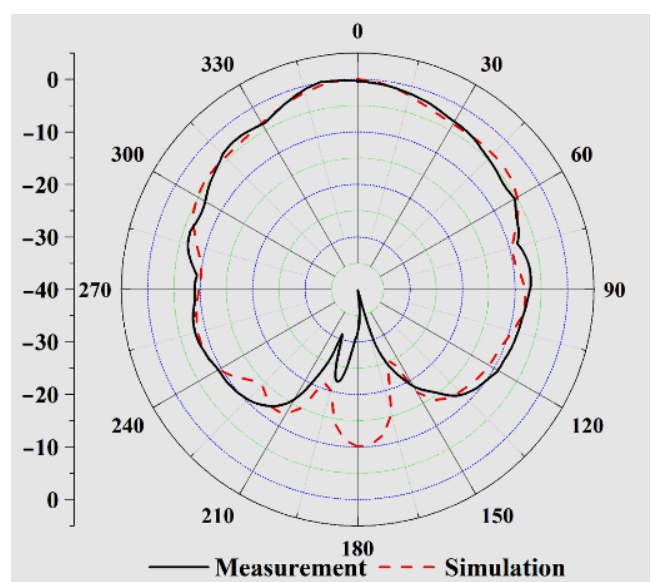

(e)

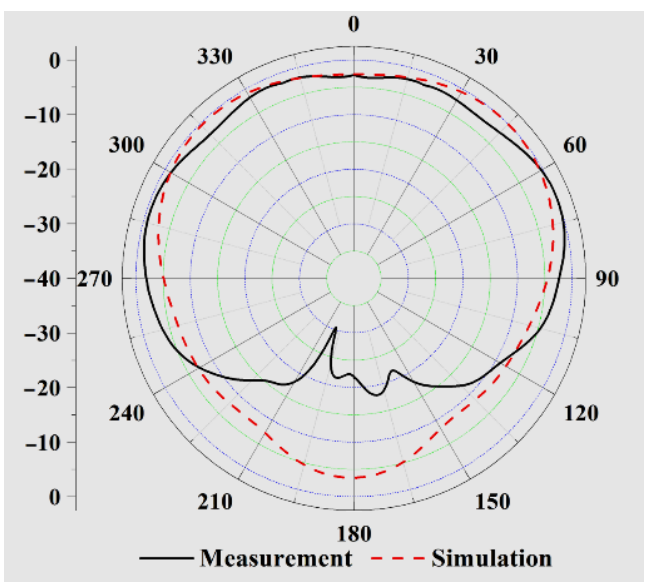

(b)

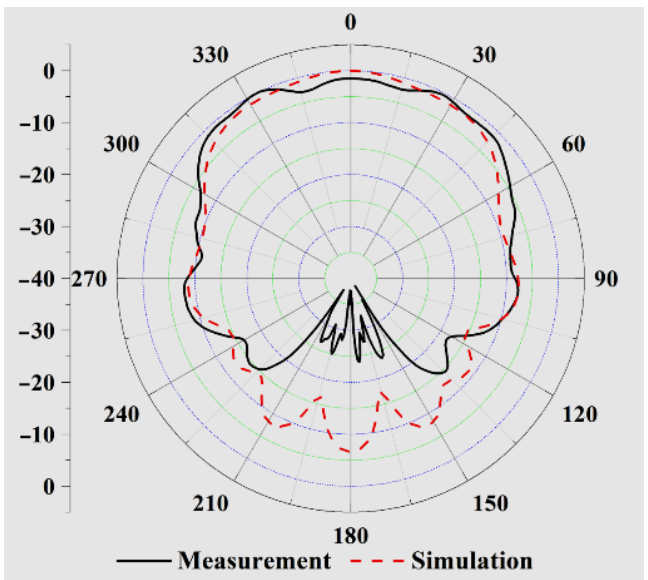

(d)

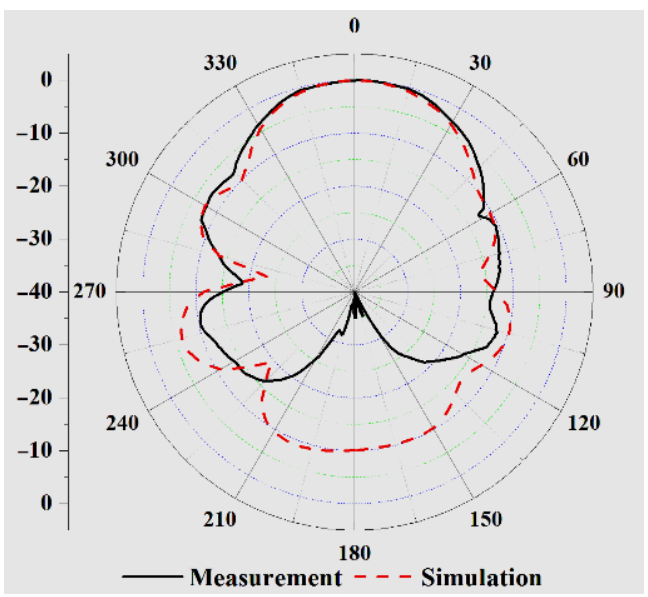

(f)

Figure 9. Measured (solid line) and simulated (dashed line) radiation patterns of the antenna in array. (a) E-plane @ 1 GHz. (b) H-plane @ 1 GHz. (c) E-plane @ 3 GHz. (d) H-plane @ 3 GHz. (e) E-plane @ $4.75 \mathrm{GHz}$. (f) H-plane @ $4.75 \mathrm{GHz}$. 


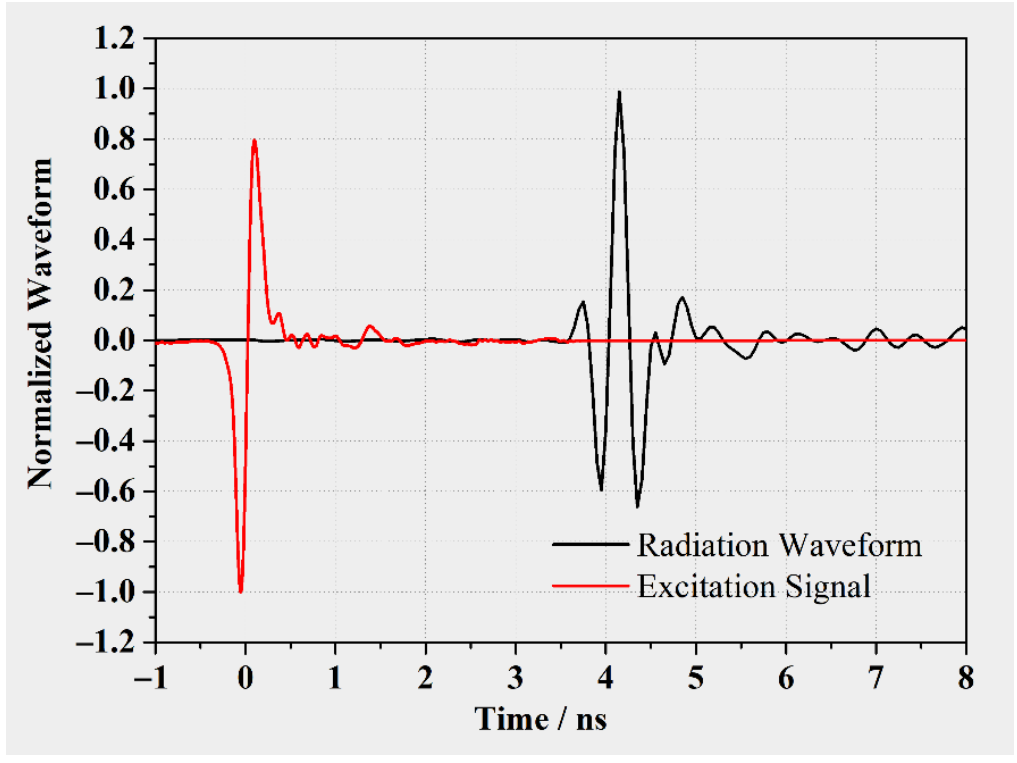

Figure 10. Radiation waveform of antenna excited by a monocycle pulse.

\section{Calibration and Experimental Validation}

It is necessary to calibrate the system to ensure the detection effect of the MIMO array. The system calibration is designed as two parts: internal calibration and external calibration. In the internal calibration, an attenuator is used to loop back the transmitting and receiving ports. By switching the switch matrix, the characteristics of delay and insertion loss of each channel are tested respectively. The signal of each channel is calibrated with the measured data, and the calibration method is shown in the Formula (3) [13]:

$$
f_{\text {internal }}(t)=a \times f_{\text {mea }}\left(t+\tau_{\text {shift }}\right)
$$

where $f_{\text {internal }}(t)$ is a signal after internal calibration, $f_{\text {mea }}(t)$ is a measured signal, $a$ is an amplitude calibration factor, $\tau_{\text {shift }}$ is a delay calibration factor.

The external calibration of the system is also needed. Strong crosstalk exists due to the short distance between antenna elements. In addition, there are complex metal structures near the array, and the multiple reflections will make the received signals messy and have a long tail. If the external calibration is not carried out, the weak reflected signals from subsurface may be masked by clutter. The core of external calibration is to remove the background signal, and the method is as Formula (4) [13]:

$$
f_{\text {external }}(t)=f_{\text {internal }}(t)-f_{\text {freespace }}(t)
$$

where $f_{\text {external }}(t)$ is the measured signal after external calibration, $f_{\text {freespace }}(t)$ is the signal in free space after internal calibration.

The signal in free space is obtained by testing in the microwave anechoic chamber. The lander and MIMO array are lifted to $1.5 \mathrm{~m}$ high. At the same time, the ground and walls are paved with absorbing materials to simulate the free space; the test status is shown in Figure 11. After calibration, the background signal caused by crosstalk and lander coupling can be removed well, while retaining the effective signal. Figure 12 shows the result of background removal. 


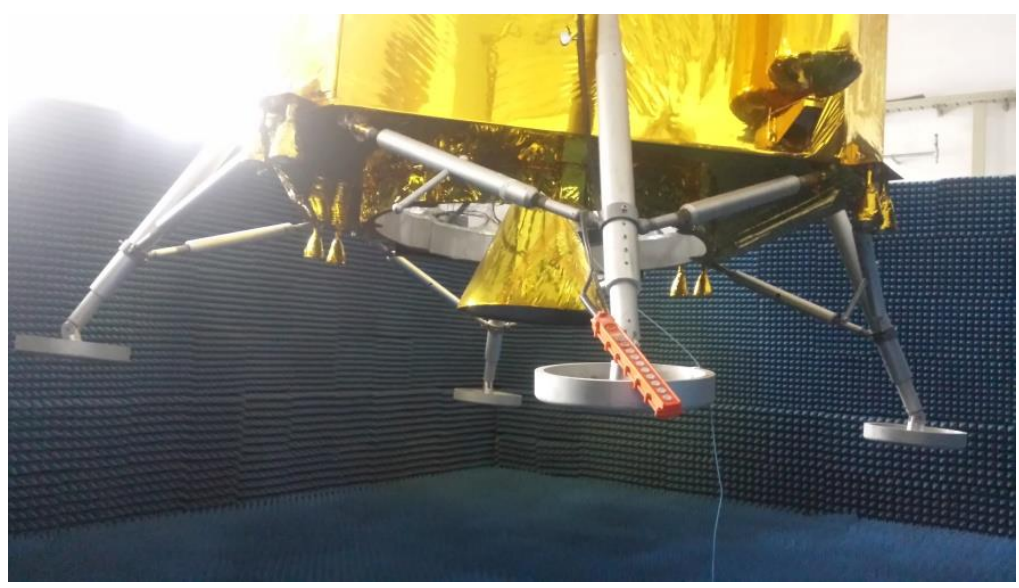

Figure 11. The external calibration status.

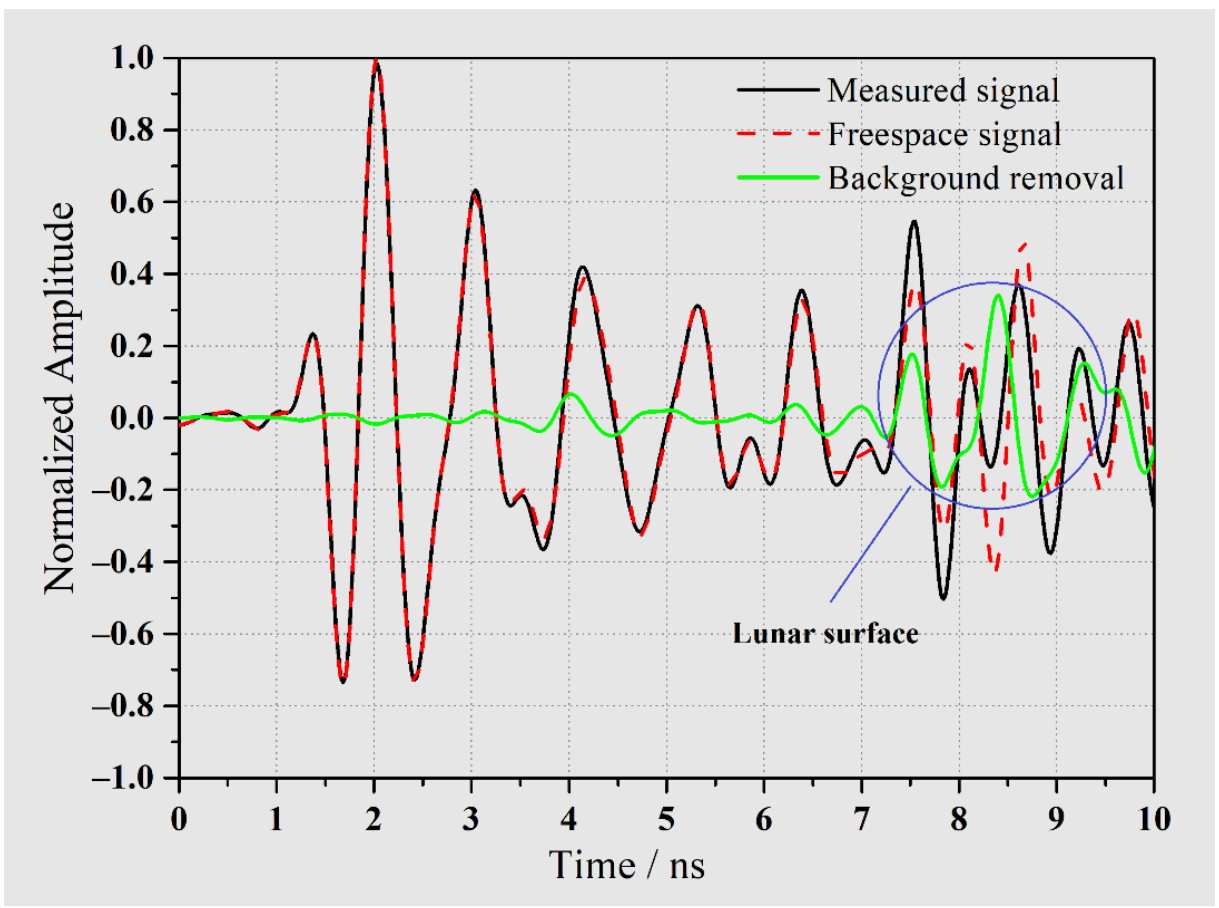

Figure 12. The results of removing the background signal.

Because the dielectric constant and loss tangent of dry volcanic ash are similar to lunar regolith, a simulated test site filled with volcanic ash with a relative permittivity of about 3 was established. The site area is $5 \times 9 \mathrm{~m}^{2}$, and a $7 \times 3 \times 2.5 \mathrm{~m}^{3}$ pool is set in the central position. In order to reduce the interference of walls and other structures, the surrounding walls are covered with absorber. Several targets at different depths, such as basalt blocks and granite slabs, are embedded in volcanic ash. The site layout and targets distribution are shown in Figure 13.

The lander was placed above the volcanic ash pool to detect embedded targets. The back projection (PB) imaging method is used in the calibration and imaging process. Before calibration, it is difficult to distinguish the locations of shallow targets in the image, as shown in Figure 14a. As displayed in Figure 14b, after internal calibration, the image quality is slightly improved, but the shallow targets are still covered by coupling signals and multiple reflection signals and cannot be identified. After the external calibration, all embedded targets can be clearly distinguished, and the pool bottom with a depth of $2.5 \mathrm{~m}$ can be clearly identified (see Figure 14c). More details of experimental validation were given by Li and Lu $[13,14]$. 


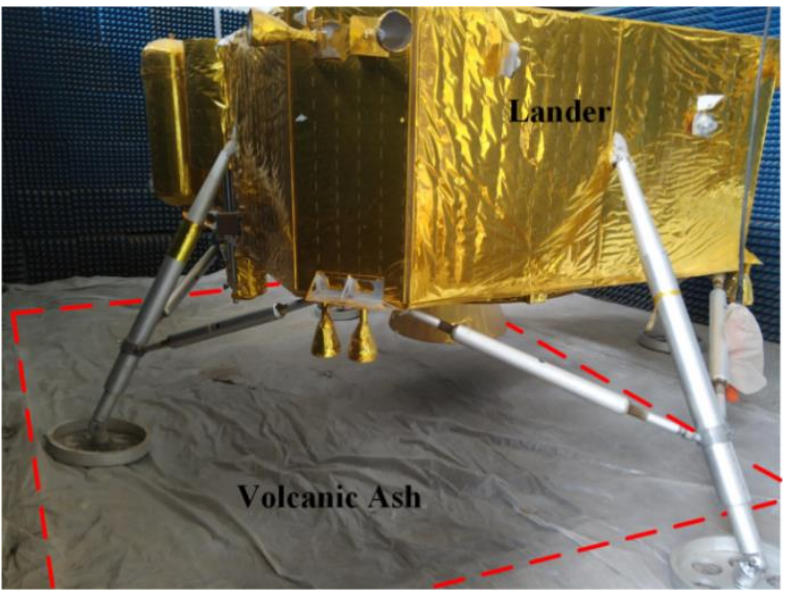

(a)

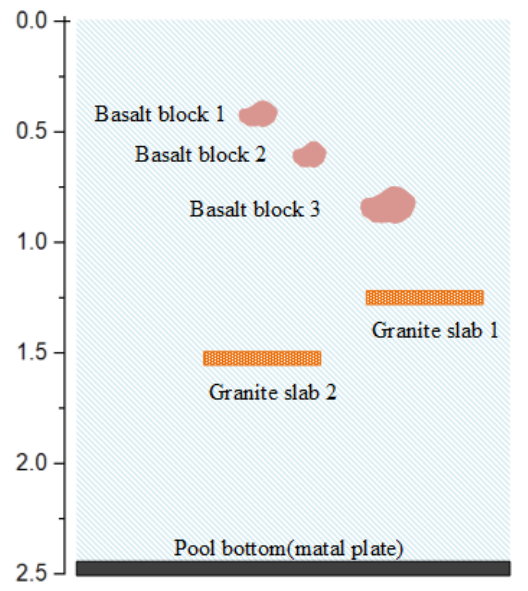

(b)

Figure 13. (a) Layout of the verification test sit. (b) Targets distribution in the volcanic ash pool.

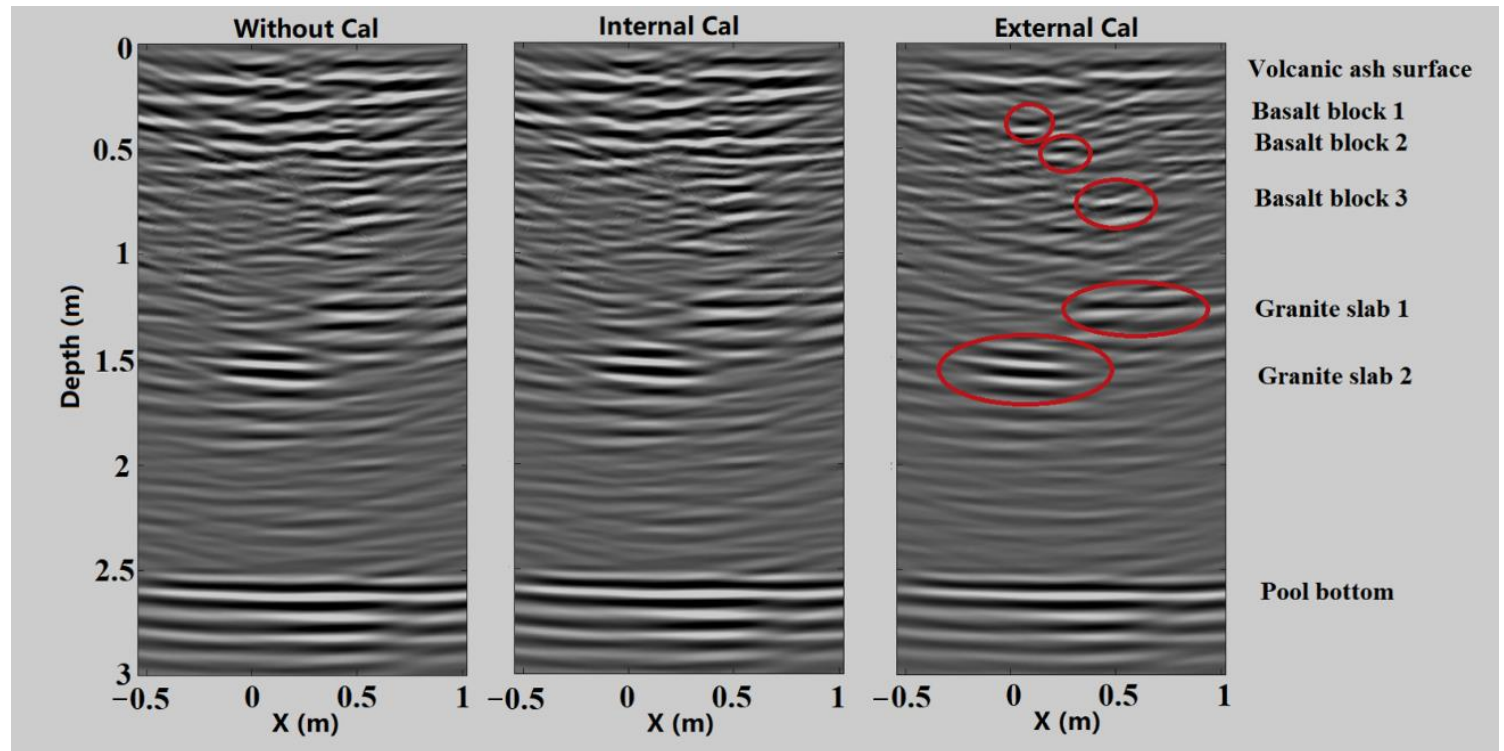

(a)

(b)

(c)

Figure 14. Results of the verification test. (a) The image before calibration; (b) the image after internal calibration; (c) the image after external calibration.

\section{Discussion}

The proposed MIMO array is suitable for stationary detection of subsurface targets and layered structures, especially for deep space exploration, such as Lunar exploration, Mars exploration and asteroid exploration, because many probes for deep space exploration are difficult to move. Due to the limitation of array length and number of elements, the detection capability of static MIMO system is slightly insufficient compared with the GPR system of mobile detection mode. However, it is still of great significance to study the subsurface structures and mineral composition of the moon and planets.

\section{Conclusions}

In this study, an ultra-wideband MIMO array capable of sub-surface probing from a stationary position was designed for the LRPR of the CE- 5 lander. The elements of the array were designed as miniaturized QESAs. Ultra-light and high temperature resistant 
radomes with high transmission rate were designed to protect the array. Through effective calibration, the MIMO array system can detect lunar regolith structures and targets within $2.5 \mathrm{~m}$ depth below the lander, which can achieve the scientific goals of LRPR. In the near future, CE-5 LRPR data will be widely available via the Data Publishing and Information Service System of China's Lunar Exploration Program (http:/ / moom.bao.ac.cn).

Author Contributions: Conceptualization, W.L. and Y.J.; methodology W.L. and Y.J.; data curation, Y.L., W.L. and B.Z.; formal analysis, W.L. and C.T.; validation, W.L., Y.L. and Y.J.; software, Y.L.; writing-original draft preparation, W.L.; writing-review and editing, Y.J. and Y.L.; visualization, W.L.; supervision, B.Z.; project administration, G.F.; funding acquisition, G.F. All authors have read and agreed to the published version of the manuscript.

Funding: This research was funded by Lunar Regolith Penetrating Radar Project for the Third Phase of Lunar Exploration Mission.

Acknowledgments: All the authors acknowledge support from the China National Space Administration. We thank the Ground Applications System of Lunar and Deep Space Exploration, National Astronomical Observatories, Chinese Academy of Science, for their efficient help in the experimental verification.

Conflicts of Interest: The authors declare no conflict of interest.

\section{References}

1. Porcello, L.J.; Jordan, R.L.; Zelenka, J.S.; Adams, G.F.; Phillips, R.J.; Brown, W.E.; Ward, S.H.; Jackson, P.L. The Apollo lunar sounder radar system. Proc. IEEE 1974, 62, 769-783. [CrossRef]

2. Kobayashi, T.; Lee, S.R.; Kumamoto, A.; Ono, T. GPR observation of the Moon from orbit: Kaguya Lunar Radar Sounder. In Proceedings of the 15th International Conference on Ground Penetrating Radar, GPR 2014, Brussels, Belgium, 30 June-4 July 2014; pp. 1037-1041.

3. Fang, G.; Zhou, B.; Ji, Y.; Zhang, Q.; Shen, S.; Li, Y.; Guan, H.; Tang, C.; Gao, Y.; Lu, W.; et al. Lunar Penetrating Radar onboard the Chang'e-3 mission. Res. Astron. Astrophys. 2014, 14, 1607-1622. [CrossRef]

4. Lai, J.; Xu, Y.; Zhang, X.; Xiao, L.; Yan, Q.; Meng, X.; Zhou, B.; Dong, Z.; Zhao, D. Comparison of Dielectric Properties and Structure of Lunar Regolith at Chang'e-3 and Chang'e-4 Landing Sites Revealed by Ground-Penetrating Radar. Geophys. Res. Lett. 2019, 46, 12783-12793. [CrossRef]

5. Zhou, B.; Li, Y.; Li, D.; Lu, W.; Shen, S.; Fang, G.; Su, Y.; Dai, S. Initial Laboratory Field Tests of the Rover-mounted GPR for China's First Mission to Mars. In Proceedings of the 2018 17th International Conference on Ground Penetrating Radar (GPR), Rapperswil, Switzerland, 18-21 June 2018.

6. Hamran, S.; Berger, T.; Brovoll, S.; Damsgård, L.; Helleren, Ø.; Øyan, M.J.; Amundsen, H.E.; Carter, L.; Ghent, R.; Kohler, J.; et al. RIMFAX: A GPR for the Mars 2020 rover mission. In Proceedings of the 2015 8th International Workshop on Advanced Ground Penetrating Radar (IWAGPR), Florence, Italy, 7-10 July 2015.

7. Zou, L.; Kikuta, K.; Sato, M. Nondestructive Inspection of an Airport Pavement by MIMO Array GPR YAKUMO. In Proceedings of the 2018 17th International Conference on Ground Penetrating Radar (GPR), Rapperswil, Switzerland, 18-21 June 2018.

8. Panzer, B. Development of an Electrically Small Vivaldi Antenna: The CReSIS Aerial Vivaldi(CAV-A). Master's Thesis, University of Kansas, Lawrence, KS, USA, 2004.

9. Lee, R.Q. NASA Tech Memorandum NASA/TM-2004-213057. Notch Antenna; Glenn Research Center: Cleveland, OH, USA, 2004.

10. Lee, K.F.; Chen, W. Advances in Microstrip and Printed Antennas. In Advances in Microstrip and Printed Antennas; Lee, K.F., Chen, W., Eds.; Publishing House: New York, NY, USA, 1997; pp. 443-514.

11. Schaubert, D.H.; Chio, T.; Holter, H. TSA element design for 50-1500 MHz array. In Proceedings of the IEEE Antennas and Propagation Society International Symposium, Salt Lake City, UT, USA, 16-21 July 2000; Volume 1, pp. 178-181.

12. Yektakhah, B. High Resolution Through-the-Wall and Subsurface Imaging Using Small Number of Transceivers on Moving Robotic Platforms. Ph.D. Thesis, University of Michigan, Ann Arbor, MI, USA, 2019.

13. Li, Y.; Lu, W.; Fang, G.; Zhou, B.; Shen, S. Performance verification of Lunar Regolith Penetrating Array Radar of Chang'E-5 mission. Adv. Space Res. 2019, 63, 2267-2278. [CrossRef]

14. Li, Y.; Lu, W.; Fang, G.; Shen, S. The Imaging Method and Verification Experiment of Chang'E-5 Lunar Regolith Penetrating Array Radar. IEEE Geosci. Remote Sens. Lett. 2018, 15, 1006-1010. [CrossRef] 